\title{
Comparative Transcriptome Profiling Analysis to Unravel the Potential Mechanism of Seed Abortion in Lumnitzera littorea
}

\author{
Jingwen Zhang ${ }^{1,2}$, Yong Yang ${ }^{2}$ and Ying Zhang ${ }^{{ }^{*}}$ \\ ${ }^{1}$ Life Science and Technology School, Lingnan Normal University, Zhanjiang 524048, P.R. China \\ ${ }^{2}$ Ministry of Education Key Laboratory for Ecology of Tropical Islands, College of Life Sciences, Hainan Normal University, \\ Haikou 571158, P.R. China \\ For correspondence: zhangyingred@yahoo.com
}

Received 20 June 2020; Accepted 29 October 2020; Published 10 January 2021

\begin{abstract}
Lumnitzera littorea (Jack) Voigt. is a species of mangrove plant belonging to the family Combretaceae. Natural reproduction of L. littorea is extremely difficult due to its seed abortion in China. To reveal the molecular mechanism of seed abortion, we performed transcriptome to analyze the seeds of $L$. littorea-T (normal seeds) and L. littorea-S (abortive seeds). After analysis of the raw data, 64,868 transcripts (mean length $=658 \mathrm{bp}$ ) were assembled. Among these transcripts, 39,779 were functionally annotated. Then, differentially expressed genes (DEGs) were screened, and 23,513 transcripts were identified that were likely involved in seed abortion. About 207 DEGs assigned to Kyoto Encyclopedia of Genes and Genomes (KEGG) pathways, and approximately $12.1 \%$ fell into reproduction categories. Genes involved in response to sucrose and starch metabolism, and phytohormone biosynthesis showed significant different expression levels between normal and abortive seeds of $L$. littorea . Further expressions patterns of key genes contribute to seed development were analyzed using quantitative real-time PCR, and the results were consistent with high-throughput sequencing data. The information obtained in this study will aid in the research of the mechanism of seed abortion in further molecular studies of L. littorea. (C) 2021 Friends Science Publishers
\end{abstract}

Keywords: Endangered mangrove; Lumnitzera littorea; Transcriptome; Seed abortion

\section{Introduction}

Mangroves are a group of woody plants that grow in tropical and sub-tropical intertidal zones. As the most productive and diverse wetlands in the coasts, mangroves provide important ecological services for costal ecosystems (Tomlinson 1986). In recent years, due to human activities and environmental changes, mangrove areas have been decreasing sharply, facing the biodiversity loss on ecosystems (Lovelock et al. 2015).

Lumnitzera littorea (Jack) Voigt. is a thermophilic mangrove species distributed in tropical Asia and Australia and listed as an endangered species in "International Convention on Wetlands" (Polidoro et al. 2010). Because of its specific living environment requirements, such as average temperatures of $21-25^{\circ} \mathrm{C}, 0.5-2.7 \%$ salinity, and light, L. littorea is only distributed in Sanya of Hainan Province and the number of individuals has decreased rapidly in the past decade, from 359 in 2006 (Fan and Chen 2006) to nine with one population in 2017 (Zhang et al. 2017). Currently, the extremely long dormancy periods and high abortion ratio of its seeds were seriously restricted sexual reproduction of L. littorea (Zhang et al. 2017).
Generally, each flower contains 3-5 ovules. However, the rate of aborted seeds is up to $76.54 \pm 0.50 \%$, and only any well-developed seed could be found in L. littorea fruit in China (Zhang et al. 2013). Owing to its rigorous ecotope demand and low fecundity, L. littorea cannot realize the natural reproduction and is critically endangered in China, while there are no reports about the natural reproduction in other countries ( $\mathrm{Su}$ et al. 2007).

In order to find the reason of seed abortion, several studies have carried out in L. littorea (Zhang et al. 2016, 2017). L. littorea is outcrossing with partial self-pollination in China. Due to the rare population, L. littorea is forced to self-pollination leading to ovule browning, pistil abortion, embryogenesis arrest and other phenomena. Second, the pollens vitality was lower than $10 \%$. Third, the embryo has been eaten by a small grub that originates from eggs laid by the parent insect early in development.

Seed abortion is common in plants and has been widely studied. Many studies focus on gene regulation to illustrate seed abortion of model plant, while there are few reports for non-model woody plants, especially for endangered tree species. Based on the de novo sequencing technology, large-scale transcriptome data were being used

To cite this paper: Zhang J, Y Yang, Y Zhang (2021). Comparative transcriptome profiling analysis to unravel the potential mechanism of seed abortion in Lumnitzera littorea. Intl J Agric Biol 25:420-426 
to establish the unigene library for non-model species. Therefore, it is possible to widely discover the genes in different species that do not contain any reference genome information (Xu et al. 2016). For example, seed abortion has caused the dove tree (Davidia involucrata Baill.) to become an endangered species. To investigate the mechanism by which species become endangered, de novo sequencing was performed. As a result, WRKY and MYB transcription factors, laccase and receptor kinase are present that play important functions in seed abortion ( $\mathrm{Li}$ et al. 2016). As the study showed that GA application could change the antioxidant enzyme activities to effect on redox homeostasis by regulating the transcript levels of various genes believed to be involved in seed development (Cheng et al. 2013, 2015).

In the present study, two materials, normal and abortive seeds of L. littorea from two different locations, Thailand and China, were used to study the mechanism of seed abortion. The transcriptomes from developed seeds of the normal and abortive materials were performed. And the differentially expressed genes (DEGs) were identified between normal and abortive seeds. The objectives of this study were to identify functional genes involved in seed abortion and highlight the molecular mechanisms related to L. littorea seed abortion in China.

\section{Materials and Methods}

\section{Sample collection}

Two types of seeds, normal and abortive seeds at the same developmental stage, were collected from two different locations. The normal seeds of $L$. littorea were collected from different trees of the naturally distributed $L$. littorea population in Lam Ri-ngun, Chanthaburi, east coast, Thailand $\left(\mathrm{N}: 12^{\circ} 23^{\prime} / \mathrm{E}: 102^{\circ} 16^{\prime}\right)$. The abortive seeds were collected in the Hainan SanYaTieLu By National Nature Reserve Administration Bureau, Hainan Province, China (P.R.). The seeds were collected approximately one month after pollination. The seeds were dissected immediately from the fruits to distinguish normal or abortive seeds after collection. The normal seeds and abortive seeds were separated and kept in RNALOCKER (TIANDZ, China).

\section{RNA extraction and sequencing}

Total RNA was extracted from the two types of young seeds (200 seeds/type). We used TRIzol reagent (Invitrogen, U.S.A.) for RNA isolation. Each digital gene expression (DGE) library contained two biological replicates. After extraction of total RNAs of each sample, qualified RNA samples were used to construct complementary DNA (cDNA) libraries with NEBNext巴 ${ }^{\circledR}$ Ultra $^{\mathrm{TM}}$ RNA Library Prep Kit (NEB, U.S.A.). The raw data, including four libraries, were uploaded to NCBI SRA (accession number: SRP115695).

\section{Transcriptome de novo assembly}

The two transcriptome libraries were sequenced. After sequencing, the raw image data were converted into raw sequence data by base calling. Then, the raw data were treated with following steps: (1) Adaptor sequences fragments, reads with unknown sequences ' $\mathrm{N}$ ' were greater than $10 \%$, and low-quality sequences (the percentage of quality value $\leq 5$ was greater than $50 \%$ in a read) were removed. (2) These filtered reads were carried out by Trinity software with min_kmer_cov set to 2 by default and all other parameters with default values (Grabherr et al. 2011). (3) After assembling the data, the contigs were mixed together for combined analysis. The combined assembled sequences were finally used as reference sequencing data for the following gene expression analysis.

\section{Functional annotation of transcripts}

All unigenes were searched against the following public databases, $\quad \mathrm{Nr}$ (non-redundant, http://www.ncbi.nlm.nih.gov/), Swiss-Prot (http://www.expasy.ch/sprot/), KEGG (Kyoto Encyclopedia of Genes and Genomes, http://www.genome.jp/kegg/) and COG (Clusters of Orthologous Groups of proteins, http://www.ncbi.nlm.nih.gov/cog/) using the BLASTX algorithm (cut-off value of $E<1 e-5$ ). To classify the unigenes, the Blast2GO program was used to get the GO annotations (Conesa et al. 2005). WEGO software was used to perform GO functional classification for all transcripts (Ye et al. 2006).

\section{Identification of DEGs}

To identify the DEGs, the gene expression value was calculated based on the FPKM method (Mortazavi et al. 2008). The gene expression value was calculated by using the numbers of reads that were mapped to the reference assembled sequence data. After calculating the gene expression level, the DEGs were screened. We performed differential gene expression analysis using the $\mathrm{R}$ package DESeq (1.10.1) (Anders and Huber 2010). In this study, DEGs were adjusted based on both genes with $P$ value < 0.01 and $\mid \log 2$ fold change (Abortive/Normal Seeds) $\mid \geq 1$.

\section{qPCR analysis}

Total RNA was extracted and reverse transcription was performed to examine the gene expression value with quantitative real-time reverse transcription PCR (qRTPCR). qPCR reaction was performed using the SYBR premix Ex Taq kit (TaKaRa, Japan) on an ABI 7500 RealTime System. A L. littorea gene, LiActin (unigene13201), was used as endogenous reference for data normalization. The relative expression of target genes was calculated using $2^{-\Delta \Delta \mathrm{CT}}$ method (Quail et al. 2008). All experimental samples 
were repeated in triplicate. All primer pairs used for qRTPCRs were listed in Additional file 1: Table S1.

\section{Results}

\section{De novo assembly}

Two cDNA libraries of normal and abortive seeds of $L$. littorea were generated and sequenced. In total, 485,698,877 raw sequencing reads were generated from the $200 \mathrm{bp}$ insert library. After filtering, 102,164,440 clean data were obtained. Then, the clean data were used to assembly analysis. After clustering the contigs, 71,390 and 63,066 transcripts were obtained. Then, the unigene information of the two libraries was used for comparative analysis. As a result, 64,868 transcripts were obtained, and the average length was $714 \mathrm{bp}$ with an N50 of 1,180 bp. For the length distribution analysis, approximately $41.95 \%$ of the transcripts had lengths greater than $500 \mathrm{bp}$ (Table 1).

\section{Annotation of all nonredundant transcripts}

To further validate and annotate the assembled transcripts, all assembled transcripts were searched against the $\mathrm{Nr}$ and SwissProt databases by BLAST 2.2.28+ program (E-value < 1E-5, Fig. 1a). For all 64,868 transcripts, 39,779 transcripts $(61.32 \%)$ had at least one significant match to an existing gene. Using the NR database, 37,523 (57.85\%) transcripts matched sequences annotated in NR (Table S2). Among them, $8,466(22.6 \%)$ shared more than $80 \%$ similarity with an established sequence (Fig. 1b). For the species similarity analysis, all of the transcripts could be mapped to approximately 200 species; a high percentage of $L$. littorea sequences (24.7\%) were homologous to Vitis vinifera genes, followed by Amygdalus persica genes (15.4\%) and Ricinus communis genes (14.8\%) (Fig. 1c). Using the NT database, $30,671(47.28 \%)$ transcripts matched sequences annotated in NT (Table S2). A total of 23,964 transcripts (36.94\%) were mapped to SwissProt compared with the NR database (Table S2). In total, 15,082 unigenes were hit the $\mathrm{Nr}$ and SwissProt protein databases, indicating that this study produced a substantial fraction of the fertility-related genes in L. littorea.

\section{Functional classification by GO and COG}

Based on the NR annotation, a Gene Ontology (GO) analysis was conducted. In total, 29,094 transcripts were assigned to GO classes with 55 functional terms. As shown in Fig. 2, the primary category was biological process $(14,142,48.61 \%)$, followed by cellular component (10,907, $37.49 \%)$, and molecular function $(4,044,13.90 \%)$. In the biological process category, cellular process $(18,349$, $15.85 \%)$ and metabolic process $(17,488,15.10 \%)$ was prominent (Fig. S2). The results indicated that important cell activities and metabolic processes occurred in the embryos formation and development stages of L. littorea. For cellular component, cell and cell part, organelles, and membrane and membrane components accounted for about 50, 26.03 and $14.04 \%$, respectively. In the molecular function category, the major subcategories were catalytic activity $(14,216,42.93 \%)$ and binding $(13,671,41.29 \%)$. The following categories contained 3,562 transcripts, representing only $10.76 \%$. Among the 37,523 transcripts with significant similarity to the NR proteins, 13,998 transcripts could be matched to COG database (Fig. S1). In total, $25 \mathrm{COG}$ categories were assigned, and the major subcategories were general function prediction cluster $(4,384,16.63 \%)$, followed by transcription $(2,376,9.01 \%)$, recombination, replication, and repair $(2,158,8.19 \%)$.

\section{Functional classification by KEGG pathway}

In addition to COG category analysis, KEGG pathway was used to analyze all 64,868 transcripts in $L$. littorea. The results indicated that $42.26 \%(27,415)$ of transcripts were positively matched with the database and could be divided into 5 main categories in 128 KEGG pathways. Of which, metabolism represented the largest proportion $(17,363$, $63.33 \%)$, followed by general information $(5,713,20.84 \%)$, organismal systems $(1,631,5.95 \%)$, environmental information processing $(1,539,5.61 \%)$, and cellular processes $(1,169,4.26 \%)$. These results indicated that the relative active metabolic processes occurred in the gamete formation process. As shown in Table S3, the KEGG pathway metabolism contained 11 categories, including lipid metabolism, nucleotide metabolism, energy metabolism, and carbohydrate metabolism.

\section{Analysis of DEGs between normal and abortive seeds}

According to screening criteria, 23,513 DEGs were detected between the normal seeds from Thailand and abortive seeds from Sanya (Table S4). A total of 13,043 transcripts were upregulated and 10,470 transcripts were downregulated in L. littorea-S compared with $L$. littorea-T. Among the differentially expressed genes, 1,920 DEGs had no homologs in the NCBI database, 10,459 transcripts were annotated with GO terms (Fig. 2a), and 8,127 transcripts were identified in the KEGG pathway annotation (Fig. $2 b$ ). Among them, approximately $5.94 \%$ transcripts were related to plant hormone signal transduction, nitrogen metabolism, and starch and sucrose metabolism, indicating that these pathways might respond to seed formation processes.

\section{Validation of DEGs by qPCR}

qRT-PCR analyses were performed to verify RNA-seq data. Eight genes with various expression patterns, related to plant hormone signal transduction, starch biosynthesis and catabolism, and cell wall invertase, were chosen for qRTPCR analysis. As shown in Fig. 3, five genes were 
Table 1: Summary of the L. littorea transcriptome assembly

\begin{tabular}{llllll}
\hline Type & Sample & Total number & Total length (bp) & Mean length (bp) & N50 (bp) \\
\hline Contig & L. littorea-T & 174,024 & $44,746,514$ & 257 & 350 \\
& L. littorea - S & 119,090 & $40,150,743$ & 337 & 645 \\
Unigene & L. littorea - T & 71,390 & $37,175,316$ & 521 & 919 \\
& L. littorea - S & 63,066 & $39,730,396$ & 630 & 1,040 \\
& All & 64,868 & $46,291,877$ & 714 & 1,180 \\
\hline
\end{tabular}

(a) E-value Distribution

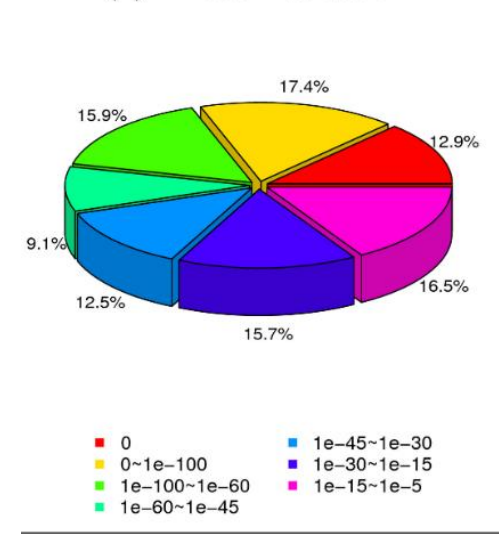

(c) Species Distribution

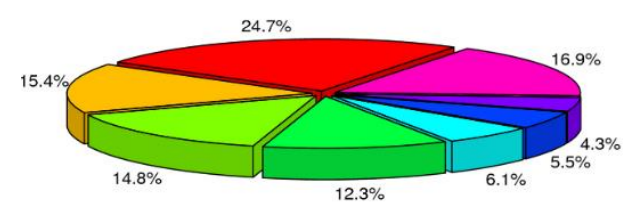

(b) Similarity Distribution

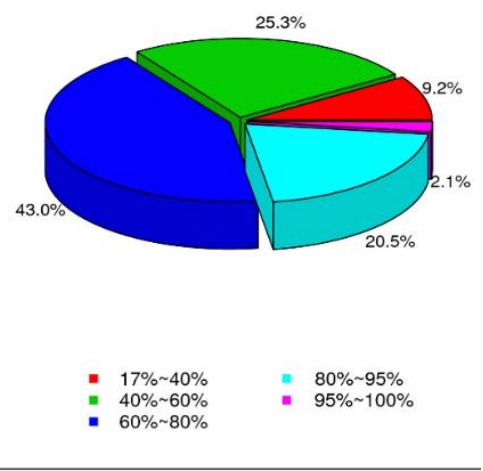

- $40 \% \sim 60 \%=95 \% \sim 100 \%$

- $60 \% \sim 80 \%$

Fig. 1: BLAST results of L. littorea transcriptome. (a) E-value and (b) Similarity distribution of the top BLAST hits. (c) Species distribution of the BLAST hits for each unigene in the NR database

significantly upregulated in abortive seeds, and the other three genes were downregulated in abortive seeds. The gene expression pattern was similar between the DGE profile and qRT-PCR, indicating that the sequencing libraries were truly representative of the differentially expressed genes between the normal and abortive seeds from the two different locations. Moreover, these genes may be target genes that cause seed abortion and require further validation.

\section{Discussion}

Being an important and endangered species of mangrove plants in China, L. littorea is a very valuable resource that has many useful genes that could be used for cultivated plant improvement. Due to its unique living environment requirements, the distribution area of L. littorea is restricted, and research is limited (Zhang et al. 2013). Most of the research has focused on cytological and tissue culture, and few molecular biology studies have investigated the mechanism of seed abortion. In this study, we analyzed the transcriptomic changes that occurred in the normal and abortive seeds of $L$. littorea from two different locations. As expected, numerous known genes were identified, including starch and sucrose metabolism genes, hormone signal transduction genes, and some possible new genes to be candidates for studying mechanisms involved in the abortion of L. littorea seeds in Sanya Province, China (Table S4 and S5).

In angiosperms, as the key yield components, the development of seeds and fruits has been studied for decades. These processes are energy-intensive and depend greatly on an adequate import of photo assimilates sucrose, which is produced in photosynthetically active leaves to support non-photosynthetic tissues, such as seeds, fruits and tubers. In the sucrose metabolism pathway, sucrose is often hydrolyzed by cell wall invertase (CWIN) into glucose and fructose (Ruan et al. 2012). Combined the transcriptomic and metabolomics analysis, high CWIN activity could promote fruit set by altering cell cycle and cell wall synthesis ( $\mathrm{Ru}$ et al. 2017). In tomato, the activity of a major CWIN gene, LIN5, is significantly increased after pollination in comparison with an unpollinated control (Shen et al. 2019). Interestingly, genes (Unigene11647_All) encoding cell wall invertase were downregulated in the abortive seeds in our study, suggesting 
(a)

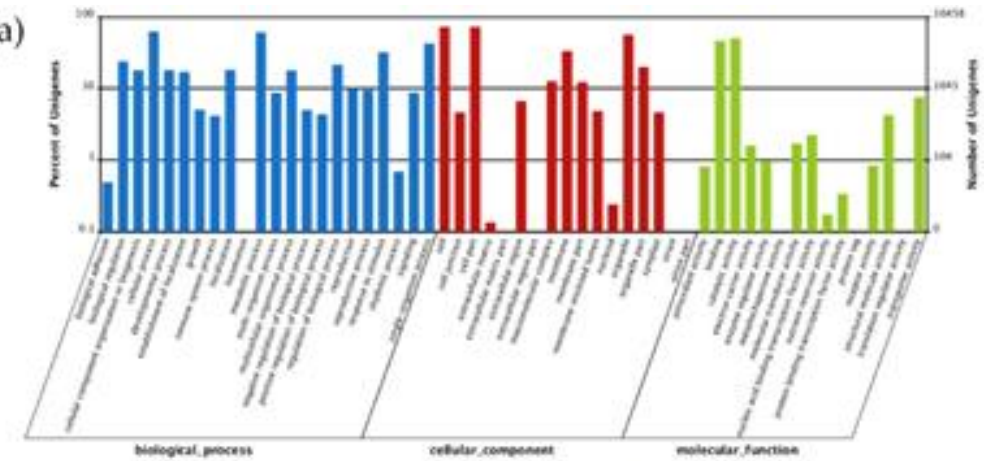

(b)

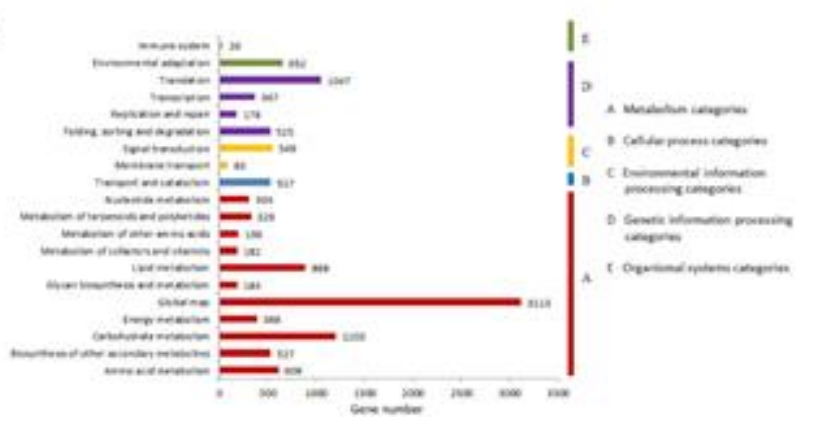

Fig. 2: Proportions of DEG transcripts by GO and KEGG. (a) GO classification of DEGs; (b) KEGG pathway of DEGs between normal and abortive seeds. The left $y$-axis indicates the number of transcripts in that main category. The bottom $x$-axis indicates the specific category of transcripts in that main category
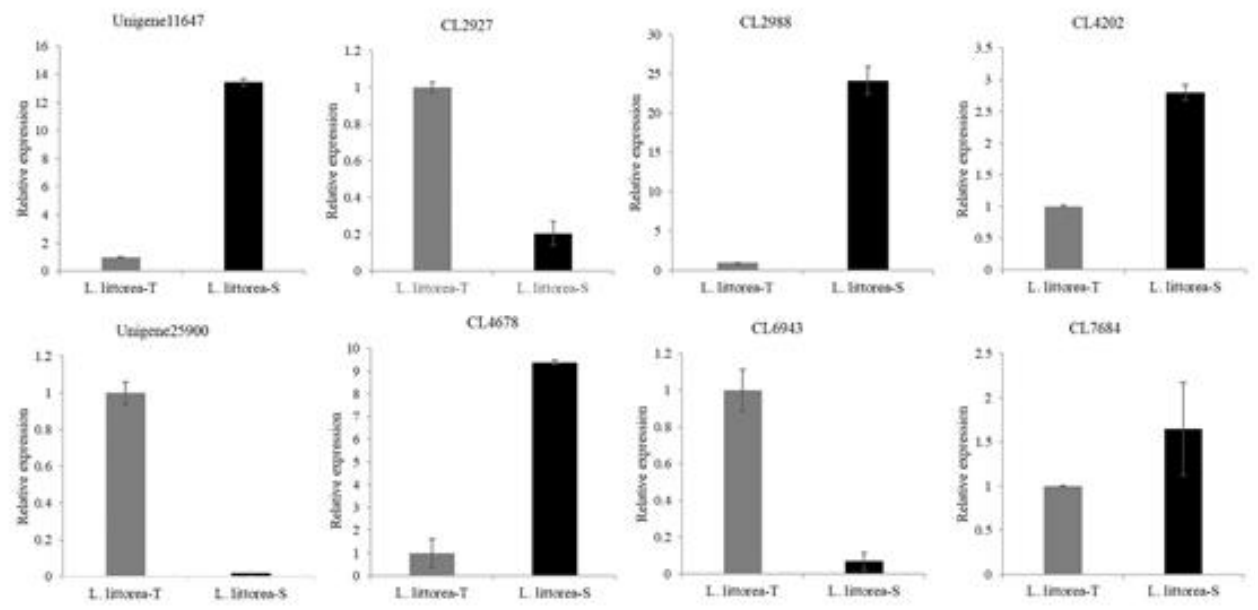

Fig. 3: Expression patterns of DEGs in L. littorea seeds. The unigene13201 was used as an internal reference. Bar depict SD (n=3)

that cell wall invertase activity deficiency may cause the seed abortion in L. littorea.

Starch, as another energy source, can be hydrolyzed into glucose, particularly under environmental stress. The resultant glucose leads to a inhibition in programmed cell death (PCD) genes and promotion in cell division, which together lead to seed and fruit set (Ruan et al. 2012). Some genes involved in starch biosynthesis and catabolism, including catalase, starch branching enzyme and fructokinase, were downregulated in $L$. littorea-S, suggesting that the starch content in abortive seed is low.
These findings provide insights into the roles of sucrose activation in fruit and seed set and identify new genetic targets to improve reproductive success.

Previous studies have shown that seed and fruit development is closely related to phytohormone regulation. The normal development of seed and fruit requires a variety of hormones, such as auxins, GAs, ethylene and brassinolides (Sun et al. 2010).

Research shows that postfertilization accumulation of auxin is required to initiate endosperm development, even in woody plants (Sun et al. 2017). Dove tree (D. involucrata), 
as an endangered species, has several genes encoding auxinresponse factors that are downregulated in abortive seeds based on comparative transcriptomics ( $\mathrm{Li}$ et al. 2016). According to our data, auxin-response factors were found among the DEGs. A number of auxin-response factors were upregulated in normal seeds. Gibberellin is a key player in fruit initiation, and GA biosynthesis genes are upregulated after pollination (Serrani et al. 2008). There were nine genes encoding gibberellin receptors among the DEGs, of which five and four gibberellin receptors were up- and downregulated, respectively. Study showed that ethylene biosynthesis and ethylene signaling genes down-regulate after pollination (Ruan et al. 2012). In our data, most ethylene-responsive transcription factors were least expressed in abortive seeds. Due to the limited samples, the DEGs involved in phytohormone biosynthesis were not as obvious as reported. Therefore, more detailed samples and sequencing should be collected for analysis.

\section{Impact of genetic diversity and population on $L$. littorea}

Genetic diversity plays an important role in allowing individual species to resist climate change (Ravenscroft et al. 2015). In endangered Acer yangbiense, a high selfing rate in seedlings was found, resulting in a low level of genetic diversity (Yang et al. 2015, 2019). The population decline in the critically endangered Ostrya rehderiana has resulted in self-pollination and seed abortion, which has caused extensive homozygosity and increased genetic load (Yang et al. 2018a). Studies comparing genomic patterns of diversity between the endangered Ostrya rehderiana (IUCN Red List) and the widespread $O$. chinensis show that $O$. rehderiana accumulates more deleterious mutations than $O$. chinensis (Yang et al. 2018b). According to our investigation, L. littorea is forced to undergo selfing in China, resulting in breeding difficulties and reduced genetic diversity in Sanya Province, China (Su et al. 2007; Zhang et al. 2017). The patterns detected in $L$. littorea may be similar to those detected in $O$. rehderiana, which requires detailed genomic information.

Plant populations are often adapted to their local environments (Leimu and Fischer 2008). Rubisco catalyses a rate-limiting step in photosynthesis and have long been a target for improvement due to its slow turnover rate (Sharwood 2017). However, the overexpression of the Rubisco assembly chaperone RUBISCO ASSEMBLY FACTOR 1 (RAF1) resulted in a $>30 \%$ increase in Rubisco content, which could improve the tolerance of maize to extreme growth environments (Salesse-Smith et al. 2018). Furthermore, Rubisco activase could regulate the activity of Rubisco and keep Rubisco in a high activation level under in vivo conditions (Bracher et al. 2017; Zhang et al. 2019). L. littorea grows in harsh environments and in ecosystems that are highly fragile and found in very limited areas in China (Zhang et al. 2017). Interestingly, three genes (Unigene36992, Unigene41498, and Unigene17234) encoding Rubisco activase were found among the DEGs, all of which showed dramatically increased expression in abortive seeds according to our research. This result showing no difference with the finding that the reduction in severely deleterious recessive variations may have allowed endangered $O$. rehderiana to survive at low population sizes over extended time periods (Yang et al. 2018a, b). Thus, further research should focus on designing an appropriate hybrid strategy to avoid inbreeding and increasing the genetic diversity rather than improving the total number of seedings through the collection of inbred seeds. A large number of mangrove plants are currently dying out, and the same strategy should be carried out to facilitate population recovery.

\section{Conclusion}

In the current study, two cDNA libraries of developing seeds from Sanya and Thailand were evaluated via highthroughput sequencing. After combined sequence assembly, 64,868 unigenes were identified, and GO, KEGG, and COG analyses were performed with these unigenes. In the DEG analysis, 23,513 DEGs were discovered, including sucrose and starch metabolism and phytohormone biosynthesis genes, which are potentially involved in seed development. Furthermore, the living environments, distribution and genetic diversity also affect the seed reproduction of $L$. littorea. Our study provides a foundation to understand and further unravel the molecular mechanism of seed abortion of L. littorea in China.

\section{Acknowledgments}

This work was funded by the National Science Foundation of Hainan Province, China (418QN240) and National Natural Science Foundation of China (Nos. 41776148).

\section{Author Contributions}

Ying Zhang designed the experiments and reviewed drafts of the paper. Jingwen Zhang analyzed the data, performed the experiments and wrote the paper. Yong Yang contributed materials.

\section{References}

Anders S, W Huber (2010). Differential expression analysis for sequence count data. Genom Biol 11; Article R106

Bracher A, SM Whitney, FU Hartl, M Hayer-Hartl (2017). Biogenesis and metabolic maintenance of rubisco. Annu Rev Plant Biol 68:29-60

Cheng C, C Jiao, SD Singer, M Gao, X Xu, Y Zhou, Z Li, Z Fei, Y Wang, $X$ Wang (2015). Gibberellin-induced changes in the transcriptome of grapevine (Vitis labrusca $\times V$. vinifera) $\mathrm{cv}$. Kyoho flowers. BMC Genomics 16; Article 128

Cheng C, X Xu, SD Singer, J Li, H Zhang, M Gao, L Wang, J Song, X Wang (2013). Effect of $\mathrm{GA}_{3}$ treatment on seed development and seed-related gene expression in grape. PLoS One 8; Article e80044 
Conesa A, S Götz, JM García-Gómez, J Terol, M Talón, M Robles (2005). Blast2GO: A universal tool for annotation, visualization and analysis in functional genomics research. Bioinformatics 21:3674-3676

Fan H, L Chen (2006). Current distribution of endangered mangrove Lumnitzera littorea (Jack.) Voigt in China. Guangxi Sci 13:226-227 Grabherr M, B Haas, M Yassour, Z Levin, DA Thompson, I Amit, X Adiconis, L Fan, R Raychowdhury, QD Zeng, ZH Chen, E Mauceli, N Hacohen, A Gnirke, N Rhind, F Palma, BW Birren, C Nusbaum, K Lindblad-Toh, N Friedman, A Regev (2011). Full-length transcriptome assembly from RNA-Seq data without a reference genome. Nat Biotechnol 29:644-652

Leimu R, M Fischer (2008). A meta-analysis of local adaptation in plants. PLoS One 3; Article e4010

Li M, X Dong, J Peng, W Xu, R Ren, J Liu, F Cao, Z Liu (2016). De novo transcriptome sequencing and gene expression analysis reveal potential mechanisms of seed abortion in dove tree (Davidia involucrata Baill.). BMC Plant Biol 16; Article 82

Lovelock CE, DR Cahoon, DA Friess, GR Guntenspergen, KW Krauss, R Reef, K Rogers, ML Saunders, F Sidik, A Swales, N Saintilan (2015). The vulnerability of Indo-Pacific mangrove forests to sealevel rise. Nature 526:559-563

Mortazavi A, BA Williams, K McCue, L Schaeffer, B Wold (2008). Mapping and quantifying mammalian transcriptomes by RNA-Seq Nat Meth 5:621-628

Polidoro BA, KE Carpenter, L Collins, NC Duke, AM Ellison, JC Ellison, EJ Farnsworth, ES Fernando, K Kathiresan, NE Koedam, SR Livingstone, SG Salmo, JC Sanciangco, S Sukardjo, Y Wang, JWH Yong (2010). The loss of species: Mangrove extinction risk and geographic areas of global concern. PLoS One 5; Article e10095

Quail MA, Kozarewa, I Smith, F Quail MAI Kozarewa, F Smith, A Scally, PJ Stephens, R Durbin (2008). A large genome center's improvements to the Illumina sequencing system. Nat Meth 5:1005-1010

Ravenscroft CH, R Whitlock, JD Fridley (2015). Rapid genetic divergence in response to 15 years of simulated climate change. Glob Change Biol 21:4165-4176

Ru L, S Osorio, L Wang, AR Fernie, JW Patrick, YL Ruan (2017). Transcriptomic and metabolomics responses to elevated cell wal invertase activity during tomato fruit set. J Exp Bot 68:4263-4279

Ruan YL, JW Patrick, M Bouzayen, S Osorio, AR Fernieet (2012), Molecular regulation of seed and fruit set. Trends Plant Sci 17:656-665

Salesse-Smith CE, RE Sharwood, FA Busch, J Kromdijk, V Bardal, DB Stern (2018). Overexpression of rubisco subunits with RAF1 increases rubisco content in maize. Nat Plants 4:802-810

Serrani JC, O Ruiz-Rivero, MA Fos (2008). Auxin-induced fruit-set in tomato is mediated in part by gibberellins. Plant J 56:922-934
Sharwood RE (2017). Engineering chloroplasts to improve Rubisco catalysis: Prospects for translating improvements into food and fiber crops. New Phytol 213:494-510

Shen S, S Ma, Y Liu, S Liao, J Li, L Wu, D Kartika, HP Mock, YL Ruan (2019). Cell wall invertase and sugar transporters are differentially activated in tomato styles and ovaries during pollination and fertilization. Front Plant Sci 10; Article 506

Su GH, YL Huang, FX Tan, SH Shi (2007). Conservation genetics of Lumnitzera littorea (Combretaceae), an endangered mangrove, from the Indo-West Pacific. Mar Biol 150:321-328

Sun X, D Shantharaj, XJ Kang, M Ni (2010). Transcriptional and hormonal signaling control of Arabidopsis seed development. Curr Opin Plant Biol 13:611-620

Sun Y, C Wang, N Wang, X Jiang, H Mao, C Zhu, F Wen, X Wang, Z Lu, G Yue, Z Xu, J Ye (2017). Manipulation of auxin response factor 19 affects seed size in the woody perennial Jatropha curcas. Sci Rep 7; Article 40844

Tomlinson PB (1986). The Botany of Mangroves, pp:374-381. Cambridge University Press, Cambridge, UK

Xu J, M Zhang, G Liu, XP Yang, XL Hou (2016). Comparative transcriptome profiling of chilling stress responsiveness in grafted watermelon seedlings. Plant Physiol Biochem 109:561-570

Yang J, HM Wariss, L Tao, R Zhang, Q Yun, P Hollingsworth, Z Dao, G Luo, H Guo, Y Ma, W Sun (2019). De novo genome assembly of the endangered Acer yangbiense, a plant species with extremely small populations endemic to Yunnan Province, China. Gigascience 8; Article giz085

Yang J, LL Zhao, JB Yang (2015). Genetic diversity and conservation evaluation of a critically endangered endemic maple, Acer yangbiense, analyzed using microsatellite markers. Biochem Syst Ecol 60:193-198

Yang Y, T Ma, Z Wang, X Chen, M Zhao, MS Olson, J Liu (2018). Genomic effects of population collapse in a critically endangered ironwood tree Ostrya rehderiana. Nat Commun 9; Article 5449

Ye J, L Fang, HK Zheng, Y Zhang, J Chen, Z Zhang (2006). WEGO: A web tool for plotting GO annotations. Nucl Acids Res 34:293-297

Zhang X, C Zhong, T Yan, Y Zhang (2016). The germplasm resource rescue of endangered mangrove (Lumnitzera littorea (Jack.) Voigt) by artificial pollination. Ecol Sci 35:38-42

Zhang Y, Y Zhou, Q Sun, D Deng, H Liu, S Chen, Z Yin (2019). Genetic determinants controlling maize rubisco activase gene expression and a comparison with rice counterparts. BMC Plant Biol 19; Article 351

Zhang Y, YH Li, XN Zhang, Y Yang (2017). Flower phenology and breeding system of endangered mangrove Lumnitzera littorea (Jack.) Voigt. Chin J Appl Environ Biol 23:0077-0081

Zhang Y, C Zhong, S Li, TL Yan, W Guan (2013). Endangered species of mangrove plants: Lumnitzera littorea. For Res Manage 5:103-107 